\title{
Cost-effectiveness evaluation of quadrivalent influenza vaccines for seasonal influenza prevention: a dynamic modeling study of Canada and the United Kingdom
}

Edward W. Thommes ${ }^{1,2^{*}}$, Afisi Ismaila ${ }^{1,3}$, Ayman Chit ${ }^{4,5}$, Genevieve Meier ${ }^{6}$ and Christopher T. Bauch ${ }^{7}$

\begin{abstract}
Background: The adoption of quadrivalent influenza vaccine (QIV) to replace trivalent influenza vaccine (TIV) in immunization programs is growing worldwide, thus helping to address the problem of influenza B lineage mismatch. However, the price per dose of QIV is higher than that of TIV. In such circumstances, cost-effectiveness analyses provide important and relevant information to inform national health recommendations and implementation decisions. This analysis assessed potential vaccine impacts and cost-effectiveness of a country-wide switch from TIV to QIV, in Canada and the UK, from a third-party payer perspective.
\end{abstract}

Methods: An age-stratified, dynamic four-strain transmission model which incorporates strain interaction, transmissionrate seasonality and age-specific mixing in the population was used. Model input data were obtained from published literature and online databases. In Canada, we evaluated a switch from TIV to QIV in the entire population. For the UK, we considered two strategies: Children aged 2-17 years who receive the live-attenuated influenza vaccine (LAIV) switch to the quadrivalent formulation (QLAIV), while individuals aged $>18$ years switch from TIV to QIV. Two different vaccination uptake scenarios in children (UK1 and UK2, which differ in the vaccine uptake level) were considered. Health and cost outcomes for both vaccination strategies, and the cost-effectiveness of switching from TIV/LAIV to QIV/ QLAIV, were estimated from the payer perspective. For Canada and the UK, cost and outcomes were discounted using $5 \%$ and $3.5 \%$ per year, respectively.

Results: Overall, in an average influenza season, our model predicts that a nationwide switch from TIV to QIV would prevent $4.6 \%$ influenza cases, $4.9 \%$ general practitioner (GP) visits, $5.7 \%$ each of emergency room (ER) visits and hospitalizations, and $6.8 \%$ deaths in Canada. In the UK (UK1/UK2), implementing QIV would prevent $1.4 \% / 1.8 \%$ of influenza cases, $1.6 \% / 2.0 \%$ each of GP and ER visits, $1.5 \% / 1.9 \%$ of hospitalizations and $4.3 \% / 4.9 \%$ of deaths. Discounted incremental cost-utility ratios of $\$ 7,961$ and $£ 7,989 / £ 7,234$ per quality-adjusted life-year (QALY) gained are estimated for Canada and the UK (UK1/UK2), both of which are well within their respective cost-effectiveness threshold values.

Conclusions: Switching from TIV to QIV is expected to be a cost-effective strategy to further reduce the burden of influenza in both countries.

Keywords: Vaccination, Transmission, Cost-effectiveness, Canada, UK, Influenza, Dynamic, QIV, TIV

\footnotetext{
* Correspondence: edward.w.thommes@gsk.com

'GSK, 7333 Mississauga Road, Mississauga, ON L5N 6L4, Canada

${ }^{2}$ Department of Mathematics \& Statistics, University of Guelph, Guelph,

Ontario, Canada

Full list of author information is available at the end of the article
}

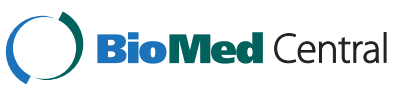

(c) 2015 Thommes et al. Open Access This article is distributed under the terms of the Creative Commons Attribution 4.0 International License (http://creativecommons.org/licenses/by/4.0/), which permits unrestricted use, distribution, and reproduction in any medium, provided you give appropriate credit to the original author(s) and the source, provide a link to the Creative Commons license, and indicate if changes were made. The Creative Commons Public Domain Dedication waiver (http://creativecommons.org/publicdomain/zero/1.0/) applies to the data made available in this article, unless otherwise stated. 


\section{Background}

Influenza A and B viruses are notable respiratory pathogens and remain an important cause of public health concern worldwide [1, 2], with annual influenza attack rates ranging from $5-10 \%$ in adults to $20-30 \%$ in children [1]. While the influenza $B$ virus has often been regarded to be milder than influenza $A$, several studies have reported that they both cause disease of similar severity, symptoms and rates of influenza-related complications [3-6]. Influenza $A$ and $B$ both cause annual epidemics in individuals of all ages, with $B$ accounting for about a quarter of cases on average, although the proportion can vary substantially from season to season, from less than $1 \%$ to over $50 \%$ [2].

Efficacious and safe influenza vaccines remain the cornerstone of influenza prevention worldwide. Until the 2012-2013 influenza season, only trivalent influenza vaccines were in use, containing two influenza A strains (A/H1N1 and $\mathrm{A} / \mathrm{H} 3 \mathrm{~N} 2)$ and only one of the two influenza $\mathrm{B}$ lineages, $\mathrm{B} /$ Victoria and $\mathrm{B} /$ Yamagata. As influenza viruses undergo frequent changes in their surface antigens, the composition of influenza vaccines is changed annually to match the circulating virus subtype expected for the next influenza season, based on the recommendations of the World Health Organization (WHO) [1, 7]. Because there is limited cross-protection between the two influenza $B$ lineages $[8,9]$, the effectiveness of each season's trivalent vaccine against influenza $B$ depends on correct prediction of the circulating $B$ lineage [10]. With co-circulation of both influenza B lineages in the last decade $[2,7,8,10,11]$, this has proven to be challenging; worldwide, the chosen $\mathrm{B}$ lineage has been mismatched to the dominant circulating lineage in about half of the seasons. As of the 2013-2014 season, the availability of quadrivalent influenza vaccines, containing both $B$ lineages each season, has offered the potential of improved protection. Indeed, vaccination with quadrivalent vaccine has shown improved immunogenicity, compared with TIV, in children, adults and elderly people [12-15].

Traditionally, annual seasonal influenza vaccination has been targeted to people classified as being at highrisk, particularly the elderly, but in recent years, with increasing evidence in favor of universal vaccination [16], vaccination recommendations have been expanded to target larger numbers and diverse population subgroups. Only some countries now recommend universal influenza immunization. In Canada, publicly-funded universal influenza immunization programs exist in all provinces except British Columbia, Quebec and New Brunswick [17]. The United Kingdom (UK) has until recently had a targeted influenza immunization program with publicly-funded immunization only for people aged 65 years and over, plus the clinical at-risk population.
However, beginning with the 2013-2014 season, rollout of a phased extension of the immunization program to healthy children has commenced $[18,19]$.

Cost-effectiveness analyses are widely used and accepted to explore and understand the impact of different strategies and interventions in diverse settings. Recently performed cost-effectiveness studies using static cohort models show that switching from TIV to quadrivalent influenza vaccine (QIV) in universal programs is a costeffective strategy [20-23]. Static models cannot however fully model the impact of plausible herd effects which are known to be afforded by vaccination [22, 24]. Moreover, the variability of model outcomes that is attributable to age-specific disease transmission parameters crucial in understanding infectious diseases has not been considered [21]. Dynamic transmission models are the preferred choice when analyzing influenza [25]. The aim of this modeling study using an age-stratified dynamic transmission disease model was to assess the public health and economic impact of a nationwide switch from TIV to QIV, in Canada and the UK, from the perspective of the healthcare provider (third party payer).

\section{Methods}

\section{Model overview}

We used a previously published compartmental (Susceptible-Infected-Recovered-Vaccinated) dynamic transmission model capturing the pairwise interactions of two influenza $\mathrm{A}$ strains, $\mathrm{A} / \mathrm{H} 1 \mathrm{~N} 1$ and $\mathrm{A} / \mathrm{H} 3 \mathrm{~N} 2$, and two influenza B lineages, B/Yamagata and B/Victoria [26]. Interactions between the strains were assumed to occur via both natural and vaccine-conferred partial crossreactive immunity. We assumed that influenza A to B cross-protection is negligible, and modeled only pairwise cross-protection between the two A strains, and between the two B lineages, respectively. The overall structure is thus of a pair of essentially independent two-strain models. Age-dependent contact patterns were specified using a contact matrix $[27,28]$ to calculate the force of infection. This model was run over a 10 -year time horizon, following a 30-year burn-in period. A detailed description of model structure, assumptions and calibration methodology are given in [26]

The model's structure makes it capable of reproducing the key transmission dynamics of seasonal influenza, specifically herd immunity, strain interaction, waning immunity and dependence on population contact patterns. A small background contribution to the force of infection (corresponding to case importation) varies randomly from season to season, (separately for influenza A and $\mathrm{B}$ ), thus rendering individual simulations stochastic. Model parameters were fit using an approximately Bayesian computation $(\mathrm{ABC})$ parameter fitting scheme [29], similar to those used previously for fitting human 
papillomavirus models $[30,31]$. The model was calibrated using, as fitting targets, the United States of America (US) unvaccinated (natural) influenza attack rates and year-to-year relative amount of influenza A compared to influenza B [21, 32]. Calibrating on only the unvaccinated population removes direct dependence of the attack rate on the efficacy and uptake of influenza vaccine in the population, though it is still coupling through herd immunity; for this reason the season-byseason vaccine uptake in the US population was also included in the calibration. Further details of the calibration process are given in [26]. The resultant posterior distribution of sets of influenza natural history parameters was then applied to the populations of two countries considered in this analyses - Canada and the UK. The detailed methodology of the calibration of this model is described in Thommes et al. [26].

\section{Model input data and assumptions}

Baseline demographic, cost, utility and vaccine-related input parameters for the dynamic transmission model were obtained from locally available databases and published literature, details of which are described below (for details see Additional file 1).

\section{Intervention strategy}

In Canada, where TIV is predominantly used in provincial or territorial public programs, we evaluated a full switch from TIV to QIV in individuals of all age groups, at the current nationwide vaccine uptake level (2014). To capture current recommendations and practice in the UK $[18,19]$, we evaluated two strategies: Children aged 2-17 years who receive the live-attenuated influenza vaccine (LAIV), while individuals of ages 18 and above receive TIV. In this analysis, the latter age group undergoes a switch from TIV to QIV and children aged 2-17 years undergo an analogous switch from the trivalent formulation of LAIV to the quadrivalent formulation of LAIV (QLAIV). Given that the pediatric vaccination program in the UK is currently in its rollout phase, two different vaccination uptake scenarios in children (denoted UK1 and UK2) were evaluated.

\section{Demographics}

Demographic data, including birth and all-cause mortality rates for Canada were based on the year 2012 and were obtained from the Statistics Canada's CANSIM online database [33]. For the UK, demographic data, birth and all-cause mortality rates were obtained from the Office of National Statistics (data based on mid-2010 population estimates) [34]. Age-dependent contact patterns specific to Canada are unavailable and hence data from the US were used [35]. For the UK, the relevant contact matrix from Mossong et al. (physical and non-physical contacts) was used [36]. The UK matrix is in terms of number of daily contacts, whereas the US matrix is in terms of daily minutes of contact; since the natural history parameter calibration was performed using the US matrix, the UK matrix had to be converted. We did this assuming a linear relationship between daily minutes and the number of contacts, with the scaling factor chosen to yield the same dominant eigenvalue for UK matrix as for the US.

\section{Outcome probabilities}

Four outcomes of symptomatic influenza were considered in this analysis - general practitioner (GP) visit, emergency room (ER) visit, hospitalization and death. In the absence of available Canada-specific outcomes probabilities, the US-derived values of Molinari et al. [37] for GP visits, hospitalization and death were used. Probability of an ER visit was derived from the probability of hospitalization using a fixed ratio between the two quantities [24]. For the UK, these outcome probabilities were obtained from Turner et al. [32] (GP visit), Tappenden et al. [38] (ER visit and hospitalization) and Meier et al. [39] (death) (Additional file 1, see Table 1.1, 1.2).

\section{Utilities}

Age-specific life-expectancy was obtained from the Life Tables and Interim Life Tables for Canada $[40,41]$ and the UK [42], respectively. Baseline utilities for Canada and the UK were obtained from Mittmann et al. [43] and Tappenden et al.[38], respectively (Additional file 1, see Table 1.3). For Canada, quality-adjusted life-year (QALY) loss per uncomplicated case and medicallyattended influenza case were obtained from Tarride et al. [24] and Sander et al. [44], respectively. For the UK, these quantities were obtained from Tappenden et al. [38] (Additional file 1, see Table 1.4).

\section{Vaccine uptake}

Vaccine uptake rates for Canada in children aged 6-23 months and 2-11 years were obtained from Moran et al. [45] and for individuals aged $\geq 12$ years from Statistics Canada's CANSIM online database [33] (Additional file 1 , see Table 1.5$)$. For the UK, individuals, clinically atrisk (ages 18-64 years and 65+ years) are vaccinated and a universal childhood vaccination program for children of ages 2-17 years is being phased in as of 2013. Given the ongoing changes in child vaccine uptake, we thus considered two scenarios (UK1 and UK2) which differ only in the vaccine uptake in children 2-17 years of age (Additional file 1, see Table 1.6).

\section{Vaccine efficacy and adverse events}

Vaccine efficacy against influenza A was assumed to be identical for both TIV and QIV. The average efficacy of 
TIV against influenza A was estimated from three Cochrane reviews in healthy children [46] adults [47] and elderly individuals [48]. This is also supported on the basis of non-inferiority data of QIV and TIV from a vaccine effectiveness study comparing both vaccines [49]. TIV efficacy against influenza B for both a lineage match and mismatch was obtained from a meta-analysis of clinical trials [8]. QIV efficacy against both lineages of influenza B was assumed to always be that of TIV against the matched $B$ lineage. Due to limitations in LAIV influenza B match-mismatch efficacy data in children of ages 3 and above [8], identical efficacies were used for matched LAIV, mismatched LAIV and QLAIV for ages 3-17 years (Additional file 1, see Table 1.7, 1.8). Vaccine-conferred protection against both influenza A and B was assumed to last only one year on average [50].

Data from clinical trials show that QIV and TIV have similar safety profiles $[13,15,49]$. Moreover, with both vaccines the occurrence of adverse events was low, and when present transient in nature [38]. Thus we excluded this parameter in the model.

\section{Costs associated with resource use}

All costs are reported in the national currencies of the two countries, i.e. the Canadian dollar (\$) and Great British Pound $(£)$. The reference year for costs was 2013. For Canada, when costs were unavailable for 2013, they were inflation-adjusted to 2013 using the Canadian Consumer Price Index [33]. In this analysis we considered the payer perspective and therefore only direct medical costs were included. Costs per GP and ER visit for Canada were obtained from Tarride et al. [24] For cost per hospitalization, data from the Canadian Institute for Health Information (CIHI) Patient Cost Estimator tool was used [51]. UK cost data was obtained using multiple sources, analogous to Van Bellinghen et al. [22] (Additional file 1, see Table 1.9).

\section{Vaccination costs}

For Canada, the blended price of TIV was estimated as an average from published sources at $\$ 6.18$ per dose [24, 44, 52]. For the UK, the price of TIV was calculated as a weighted average of all TIV available on the UK market and estimated at $£ 6.39$ per dose [53]. For QIV in the UK, the list price of $£ 9.94$ per dose was used [54]. This constitutes a TIV-QIV price difference of a factor of $~ 1.56$; a hypothetical QIV price for Canada of $\$ 9.61$ per dose was derived assuming the same relative price difference. Finally, for LAIV and QLAIV, modeled only in the UK scenarios, the list price of $£ 14.00$ per dose was used [54]. For Canada, cost of vaccine administration is taken to be at $\$ 3.78$ [44]. In the UK, vaccination was assumed to take place as part of a regular GP visit, and thus to incur no additional vaccine administration cost (Additional file 1, see Table 1.9).

\section{Analyses}

\section{Base case analyses}

Health and cost outcome measures resulting from the two vaccination strategies (QIV and TIV) and the difference between QIV and TIV are estimated. For Canada, calculations were performed using a discount rate of $5 \%$ per year for monetary and utility costs as well as outcomes [55]. In the case of the UK, a discount rate of $3.5 \%$ per year was applied to costs and outcomes [56] (see Additional file 1, Table 1.10). To determine the cost-effectiveness of implementing a switch from TIV to QIV, the incremental cost-utility ratio (ICUR) was calculated from the third party payer perspective. Costeffectiveness thresholds of \$40,000-50,000 per QALY gained [44] and $£ 20,000$ per QALY gained [57] were assumed for Canada and the UK, respectively.

To simulate the impact of a switch from TIV to QIV in Canada and the UK, we performed for each country a set of 1,000 pairs of simulations with the influenza natural history input parameters for each pair drawn from the aforementioned posterior parameter sets. Each pair consists of one simulation in which a switch from TIV to QIV occurs in the 2014-2015 season (the intervention), and another in which the use of TIV is continued (the comparator). For each simulation, results were recorded from the beginning of the 2014-2015 season to the end of the 2023-2024 season, i.e. over ten seasons. Seasonal averages of influenza infections were estimated for this time period.

\section{Deterministic sensitivity analyses}

In each scenario evaluated, the distributions of outcomes produced by our ensemble of 1,000 simulation pairs reflects both our uncertainty about the true natural history parameters of influenza, and the stochastic nature of the individual simulations. In other words, our ensemble of simulations constitutes a probabilistic sensitivity analysis (PSA) on natural history parameters of influenza as well as on the variability of influenza seasons.

We also performed a combination of univariate and multivariate sensitivity analyses to investigate the sensitivity of the model outcome, specifically, of the point estimate of cost per QALY gained (i.e. the ICUR) estimated by the model. Different parameters were varied:

- QIV price per dose (Canada and UK) to the upper and lower limits of their respective range values, see Additional file 1, Table 1.9.

- QALY loss (one-sided analysis; for the lower bound, we neglected all non-death QALY loss),

- All breakthrough infection outcomes: If vaccination reduces the severity of influenza in breakthrough infections (i.e. infections of people who are vaccinated within the current season), this has the 
potential to reduce the benefit of QIV relative to TIV. In this sensitivity analysis (multivariate and one-sided), the most extreme possible scenario was considered, wherein TIV and QIV both have $100 \%$ efficacy against all influenza-associated outcomes (GP visit, ER visit, hospitalization and death). This was done by simultaneously setting all the corresponding outcomes probabilities to zero for breakthrough infections.

- Probability of hospitalization (P_hosp), breakthrough infection: Like all breakthrough infection outcomes above, but with $100 \%$ vaccine efficacy only against hospitalization. Vaccine efficacy against all other outcomes is as in the base case, equal to the vaccine efficacy against infection.

- Outcomes probabilities: Multivariate analysis with all outcome probabilities varied simultaneously at their
$95 \%$ confidence interval (CI) lower and upper limits (see Additional file 1, Table 1.1, Table 1.2).

- Discount rate: For Canada (base case $=5.0 \%$ ) and the UK (base case $=3.5 \%$ ) one-sided sensitivity analysis using $3.5 \%$ and $5.0 \%$ discount rates, respectively, and,

- GP cost, ER cost, and hospitalization cost: For Canada and the UK to the upper and lower limits of their respective range values (see Additional file 1, Table 1.9).

\section{Results \\ Base case analyses \\ Health outcomes}

Overall, in an average influenza season, our model predicts that a nationwide switch from TIV to QIV would prevent in Canada, $4.6 \%(\mathrm{n}=135,538)$ of influenza

Table 1 Overall mean health and cost outcomes for Canada

\begin{tabular}{|c|c|c|c|c|}
\hline & TIV & QIV & Difference (TIV vs. QIV) & $\%$ Difference \\
\hline \multicolumn{5}{|c|}{ Average influenza-related outcomes per season, n (95 \% Cl) } \\
\hline Cases & $2,933,460(2,532,276 ; 3,351,695)$ & $2,797,922(2,392,853 ; 3,199,681)$ & $\begin{array}{l}-135,538(-228,154 ; \\
-76,677)\end{array}$ & $-4.6(-7.7 ;-2.7)$ \\
\hline GP visits & $1,066,568(921,034 ; 1,218,892)$ & $1,014,368(868,298 ; 1,160,118)$ & $-52,200(-88,460 ;-29,055)$ & $-4.9(-8.2 ;-2.8)$ \\
\hline ER visits & $59,704(51,257 ; 68,574)$ & $56,309(47,987 ; 64,721)$ & $-3,395(-5,907 ;-1,731)$ & $-5.7(-9.7 ;-3.0)$ \\
\hline Hospitalizations & $32,986(28,319 ; 37,886)$ & $31,110(26,512 ; 35,757)$ & $-1,876(-3,264 ;-956)$ & $-5.7(-9.7 ;-3.0)$ \\
\hline Deaths & $4,836(4,114 ; 5,606)$ & $4,508(3,811 ; 5,230)$ & $-328(-584 ;-156)$ & $\begin{array}{l}-6.8(-11.9 ; \\
-3.2)\end{array}$ \\
\hline \multicolumn{5}{|c|}{ Average costs per season, \$ $(95 \% \mathrm{Cl})$} \\
\hline Vaccination & $\$ 114,269,815(113,818,022 ; 114,795,606)$ & $\$ 153,621,770(153,014,388 ; 154,328,631)$ & \multicolumn{2}{|c|}{$\$ 39,351,954(39,196,367 ; 39,533,025)$} \\
\hline GP visits & $\$ 45,574,462(39,355,763 ; 52,083,266)$ & $\$ 43,343,948(37,102,387 ; 49,571,823)$ & \multicolumn{2}{|c|}{$-\$ 2,230,514(-3,779,889 ;-1,241,510)$} \\
\hline ER visits & $\$ 13,337,288(11,450,298 ; 15,318,666)$ & $\$ 12,578,851(10,719,802 ; 14,457,926)$ & \multicolumn{2}{|c|}{$-\$ 758,437(-1,319,645 ;-386,684)$} \\
\hline Hospitalization & $\$ 120,689,432(103,441,040 ; 138,855,265)$ & $\$ 113,695,532(96,748,864 ; 130,839,986)$ & \multicolumn{2}{|c|}{$-\$ 6,993,900(-12,204,664 ;-3,525,203)$} \\
\hline $\begin{array}{l}\text { Total payer } \\
\text { costs }\end{array}$ & $\$ 293,870,997(268,805,179 ; 320,376,234)$ & $\$ 323,240,101(298,328,487 ; 348,108,942)$ & \multicolumn{2}{|c|}{$\$ 29,369,104(22,016,160 ; 34,131,5416)$} \\
\hline \multicolumn{5}{|c|}{ Total costs, 2014-24: $5 \%$ discounted, \$ (95\% Cl) } \\
\hline Vaccination & $\$ 868,017,693(864,552,580 ; 872,000,633)$ & $\begin{array}{l}\$ 1,166,943,465(1,162,285,045 \\
1,172,298,039)\end{array}$ & \multicolumn{2}{|c|}{$\$ 298,925,772(297,732,465 ; 300,297,407)$} \\
\hline GP visits & $\$ 347,201,538(298,121,148 ; 398,164,181)$ & $\$ 329,329,994(280,856,904 ; 378,138,361)$ & \multicolumn{2}{|c|}{$\$-17,871,544(-29,416,959 ;-9,870,111)$} \\
\hline ER visits & $\$ 101,255,332(86,543,184 ; 116,554,469)$ & $\$ 95,274,727(80,828,208 ; 109,823,373)$ & \multicolumn{2}{|c|}{$\$-5,980,605(-10,120,952 ;-3,025,115)$} \\
\hline Hospitalization & $\$ 915,691,926(781,948,765 ; 1,054,610,228)$ & $\$ 860,655,203(728,830,865 ; 993,481,676)$ & \multicolumn{2}{|c|}{$\$-55,036,723(-93,315,528 ;-27,516,515)$} \\
\hline $\begin{array}{l}\text { Total payer } \\
\text { costs }\end{array}$ & $\begin{array}{l}\$ 2,232,166,489(2,035,062,286 \\
2,436,875,507)\end{array}$ & $\begin{array}{l}\$ 2,452,203,389(2,261,195,912 \\
2,649,106,709)\end{array}$ & \multicolumn{2}{|c|}{$\$ 220,036,899(166,524,687 ; 257,670,736)$} \\
\hline \multicolumn{5}{|c|}{ QALYs lost, n (95\% Cl) } \\
\hline Per season & $68,980(59,036 ; 79,436)$ & $64,930(55,206 ; 74,837)$ & \multicolumn{2}{|l|}{$-4,050(-7,076 ;-2,033)$} \\
\hline Total & $522,596(446,330 ; 601,554)$ & $490,805(414,820 ; 567,537)$ & \multicolumn{2}{|l|}{$-31,791(-54,079 ;-15,845)$} \\
\hline \multicolumn{5}{|c|}{ LYs lost, n (95 \% Cl) } \\
\hline Per season & $45,675(38,909 ; 52,852)$ & $42,732(36,152 ; 49,573)$ & \multicolumn{2}{|l|}{$-2,944(-5,215 ;-1,417)$} \\
\hline Total & $344,912(293,245 ; 398,169)$ & $322,013(270,885 ; 374,069)$ & \multicolumn{2}{|l|}{$-22,899(-39,878 ;-10,871)$} \\
\hline
\end{tabular}

Note: A negative value for the difference denotes outcomes prevented; GP, general practitioner; ER, emergency room; QALYs, quality-adjusted life years; LYs, life years 
Table 2 Overall mean health and cost outcomes for the UK

\begin{tabular}{|c|c|c|c|c|}
\hline \multicolumn{5}{|l|}{ UK1 } \\
\hline & TIV/LAIV & QIV/QLAIV & $\begin{array}{l}\text { Difference (TIV/LAIV } \\
\text { vs QIV/QLAIV) }\end{array}$ & \% Difference \\
\hline \multicolumn{5}{|c|}{ Average influenza-related outcomes per season, n (95 \% Cl) } \\
\hline Cases & $6,175,473(5,348,157 ; 7,095,022)$ & $6,086,718(5,244,706 ; 6,981,581)$ & $-88,755(-153,607 ;-46,761)$ & $-1.4(-2.5 ;-0.8)$ \\
\hline GP visits & $1,477,243(1,276,226 ; 1,698,398)$ & $1,454,327(1,249,078 ; 1,672,087)$ & $-22,917(-39,758 ;-12,127)$ & $-1.6(-2.7 ;-0.8)$ \\
\hline ER visits & $45,688(39,471 ; 52,528)$ & $44,979(38,631 ; 51,714)$ & $-709(-1,230 ;-375)$ & $-1.6(-2.7 ;-0.8)$ \\
\hline Hospitalizations & $71,740(62,217 ; 82,455)$ & $70,690(61,026 ; 81,103)$ & $-1,050(-1,824 ;-553)$ & $-1.5(-2.5 ;-0.8)$ \\
\hline Deaths & $5,366(4,609 ; 6,207)$ & $5,136(4,372 ; 5,941)$ & $-230(-424 ;-107)$ & $-4.3(-7.5 ;-2.0)$ \\
\hline \multicolumn{5}{|c|}{ Average costs per season, $£(95 \% \mathrm{Cl})$} \\
\hline Vaccination & $£ 142,869,897(142,132,194 ; 143,838,565)$ & $£ 173,690,646(172,847,546 ; 174,801,973)$ & \multicolumn{2}{|c|}{$£ 30,820,749(30,714,458 ; 30,964,151)$} \\
\hline GP visits & $£ 54,658,003(47,220,348 ; 62,840,730)$ & $£ 53,810,083(46,215,893 ; 61,867,225)$ & \multicolumn{2}{|l|}{$-£ 847,920(-1,471,032 ;-448,693)$} \\
\hline ER visits & $£ 6,167,872(5,328,571 ; 7,091,250)$ & $£ 6,072,188(5,215,223 ; 6,981,395)$ & \multicolumn{2}{|l|}{$-£ 95,683(-165,998 ;-50,633)$} \\
\hline Hospitalization & $£ 316,143,107(273,230,094 ; 363,500,069)$ & $£ 310,054,168(266,192,382 ; 356,545,209)$ & \multicolumn{2}{|c|}{$-£ 6,088,939(-10,684,477 ;-3,156,736)$} \\
\hline $\begin{array}{l}\text { Total payer } \\
\text { costs }\end{array}$ & $£ 519,838,878(469,625,943 ; 576,456,567)$ & $£ 543,627,085(491,853,288 ; 598,787,408)$ & \multicolumn{2}{|c|}{$£ 23,788,206(18,451,500 ; 27,182,411)$} \\
\hline \multicolumn{5}{|c|}{ Total costs, 2014-24: $3.5 \%$ discount, $£(95 \%$ Cl) } \\
\hline Vaccination & $\begin{array}{l}£ 1,182,629,311(1,176,522,193 ; \\
1,190,629,232)\end{array}$ & $\begin{array}{l}£ 1,438,387,119(1,431,392,398 \\
1,447,571,973)\end{array}$ & \multicolumn{2}{|c|}{$£ 255,757,808(254,873,033 ; 256,946,194)$} \\
\hline GP visits & $£ 449,966,551(390,148,320 ; 516,657,013)$ & $£ 442,621,741(381,366,507 ; 508,088,148)$ & \multicolumn{2}{|c|}{$-£ 7,344,811(-12,406,117 ;-3,925,542)$} \\
\hline ER visits & $£ 50,776,387(44,026,211 ; 58,302,059)$ & $£ 49,947,563(43,035,229 ; 57,335,107)$ & \multicolumn{2}{|l|}{$-£ 828,824(-1,399,966 ;-442,977)$} \\
\hline Hospitalization & $\begin{array}{l}£ 2,603,109,409(2,257,958,720 ; \\
2,989,825,442)\end{array}$ & $\begin{array}{l}£ 2,550,795,544(2,197,010,408 ; \\
2,929,281,052)\end{array}$ & \multicolumn{2}{|c|}{$-£ 52,313,865(-89,406,033 ;-26,864,867)$} \\
\hline $\begin{array}{l}\text { Total payer } \\
\text { costs }\end{array}$ & $\begin{array}{l}£ 4,286,481,659(3,871,840,486 ; \\
4,753,225,888)\end{array}$ & $\begin{array}{l}£ 4,481,751,967(4,063,534,469 ; \\
4,933,835,213)\end{array}$ & \multicolumn{2}{|c|}{$£ 195,270,308(152,116,034 ; 224,188,224)$} \\
\hline \multicolumn{5}{|c|}{ QALYs lost, n (95\% Cl) } \\
\hline Per season & $138,681(119,774 ; 159,443)$ & $135,415(116,128 ; 155,866)$ & \multicolumn{2}{|l|}{$-3,266(-5,749 ;-1,616)$} \\
\hline Total & $1,143,215(990,661 ; 1,314,381)$ & $1,115,337(958,023 ; 1,283,316)$ & \multicolumn{2}{|l|}{$-27,878(-48,670 ;-13,753)$} \\
\hline \multicolumn{5}{|c|}{ LYs lost, n (95\% Cl) } \\
\hline Per season & $67,761(58,243 ; 78,145)$ & $65,340(55,700 ; 75,446)$ & \multicolumn{2}{|l|}{$-2,421(-4,407 ;-1,150)$} \\
\hline Total & $560,869(482,171 ; 647,615)$ & $540,396(459,999 ; 623,573)$ & \multicolumn{2}{|l|}{$-20,473(-37,239 ;-9,741)$} \\
\hline
\end{tabular}

UK2
TIV/LAIV
QIV/QLAIV

Difference (TIV/LAIV vs QIV/ \% Difference QLAIV)

Average influenza-related outcomes per season, n (95 \% Cl)

$\begin{array}{lll}\text { Cases } & 5,335,431(4,663,367 ; 6,094,108) & 5,237,644(4,563,870 ; 5,976,162) \\ \text { GP visits } & 1,279,954(1,116,137 ; 1,463,587) & 1,254,916(1,091,268 ; 1,432,823) \\ \text { ER visits } & 39,586(34,520 ; 45,266) & 38,812(33,751 ; 44,314) \\ \text { Hospitalizations } & 61,812(54,111 ; 70,575) & 60,658(52,902 ; 69,153) \\ \text { Deaths } & 4,772(4,146 ; 5,483) & 4,538(3,921 ; 5,202)\end{array}$

$$
\begin{array}{ll}
-97,787(-167,789 ;-53,233) & -1.8(-3.0 ;-1.0) \\
-25,038(-42,527 ;-13,756) & -2.0(-3.2 ;-1.1) \\
-774(-1,315 ;-425) & -2.0(-3.2 ;-1.1) \\
-1,154(-1,992 ;-626) & -1.9(-3.1 ;-1.1) \\
-234(-410 ;-116) & -4.9(-8.3 ;-2.5)
\end{array}
$$

Average costs per season, $£(95 \% \mathrm{Cl})$

$\begin{array}{ll}\text { Vaccination } & £ 171,724,730(170,737,027 ; 172,880,105) \\ \text { GP visits } & £ 47,358,314(41,297,066 ; 54,152,709) \\ \text { ER visits } & £ 5,344,140(4,660,159 ; 6,110,852) \\ \text { Hospitalization } & £ 274,233,102(239,080,248 ; 313,959,886) \\ \begin{array}{l}\text { Total payer } \\ \text { costs }\end{array} & £ 498,660,286(457,399,436 ; 545,711,073)\end{array}$

$£ 202,522,845(201,431,528 ; 203,801,765)$ $£ 46,431,902(40,376,915 ; 53,014,460)$

$£ 30,798,116(30,696,168 ; 30,920,847)$ $£ 5,239,599(4,556,325 ; 5,982,406)$ $-£ 926,412(-1,573,508 ;-508,968)$ $-£ 104,541(-177,562 ;-57,434)$ $£ 267,724,197(232,618,317 ; 305,657,406)$ $-£ 6,508,906(-11,183,958 ;-3,502,201)$ $£ 521,918,543(480,857,526 ; 567,317,462)$ $£ 23,258,257(17,987,595 ; 26,732,485)$ 
Table 2 Overall mean health and cost outcomes for the UK (Continued)

\begin{tabular}{llll}
\hline \multicolumn{2}{l}{ Total costs, $2014-24,3.5 \%$ discount, $£(95 \% \mathrm{Cl})$} & & \\
Vaccination & $£ 1,421085,767(1,412,898,941 ;$ & $£ 1,676,655,043(1,667,605,341 ;$ & $£ 255,569,276(254,720,544 ; 256,584,332)$ \\
& $1,430,629,196)$ & $1,687,226,238)$ & \\
GP visits & $£ 390,019,191(338,972,997 ; 445,806,414)$ & $£ 382,011,087(331,356,732 ; 436,393,837)$ & $-£ 8,008,104(-13,328,069 ;-4,445,418)$ \\
ER visits & $£ 44,011,639(38,251,341 ; 50,306,937)$ & $£ 43,107,966(37,391,885 ; 49,244,777)$ & $-£ 903,673(-1,504,003 ;-501,642)$ \\
Hospitalization & $£ 2,258,890,346(1,965,081,255 ;$ & $£ 2,203,039,151(1,911,631,217 ;$ & $-£ 55,851,195(-94,174,503 ; 30,373,049)$ \\
& $2,581,768,165)$ & $2,513,877,596)$ & \\
Total payer & $£ 4,114,006,943(3,771,593,455 ;$ & $£ 4,304,813,247(3,962,279,878 ;$ & $£ 190,806,304(147,019,450 ; 220,067,782)$ \\
costs & $4,497,105,857)$ & $4,678,534,060)$ & \\
QALYs lost, n $(95 \% \mathrm{Cl})$ & & \\
Per season & $121,040(105,258 ; 138,570)$ & $117,603(102,122 ; 134,418)$ & $-3,437(-5,970 ;-1,802)$ \\
Total & $998,215(867,812 ; 1,140,983)$ & $968,900(839,411 ; 1,106,335)$ & $-29,315(-49,909 ;-15,593)$ \\
LYs lost, n $(95 \% \mathrm{Cl})$ & & $-2,484(-4,309 ;-1,247)$ \\
Per season & $59,931(51,974 ; 68,794)$ & $57,448(49,677 ; 65,930)$ & $-20,996(-36,237 ;-10,647)$ \\
Total & $496,291(429,909 ; 568,923)$ & $475,295(409,468 ; 545,073)$ & \\
\hline
\end{tabular}

Note: A negative value for the difference denotes outcomes prevented; GP, general practitioner; ER, emergency room; QALYs, quality-adjusted life years; LYs, life years

cases, $4.9 \%(n=52,200)$ GP visits, $5.7 \%(n=3,395)$ ER visits, $5.7 \%(\mathrm{n}=1,876)$ hospitalizations and $6.8 \%(\mathrm{n}=$ 328) deaths (Table 1). In the UK1 scenario (i.e. vaccine uptake among children as of 2013), a switch from TIV to QIV is expected to prevent $1.4 \%(\mathrm{n}=88,755)$ influenza cases, $1.6 \%(n=22,917)$ GP visits, $1.6 \%(n=709)$ ER visits, $1.5 \%(n=1,050)$ hospitalizations and $4.3 \%$ (n = 230) deaths. In the UK2 scenario (higher than UK1; projected target vaccine uptake among children in the future), switching from the trivalent to the quadrivalent formulation is expected to have a slightly higher impact than that observed with the UK1 scenario. Under the assumptions of the UK2 scenario, the model predicts that a switch from the trivalent to the quadrivalent formulation would prevent $1.8 \%(\mathrm{n}=97,787)$ influenza cases, $2.0 \%(\mathrm{n}=25,028)$ GP visits, $2.0 \%(\mathrm{n}=774)$ ER visits, $1.9 \%(n=1,154)$ hospitalizations and $4.9 \%(n=234)$ deaths (Table 2).

Outcomes results are also reported in age-stratified form for age groups $0-4,5-19,20-49,50-64,65-74$, 75-84 and 85-99 years; see Additional file 2, Additional file 3. This presentation provides additional insight. For example, in Canada the percentage of deaths prevented across all ages is higher than that of cases prevented, and in the UK the difference is even more pronounced. Now the case fatality ratio for influenza i.e. probability

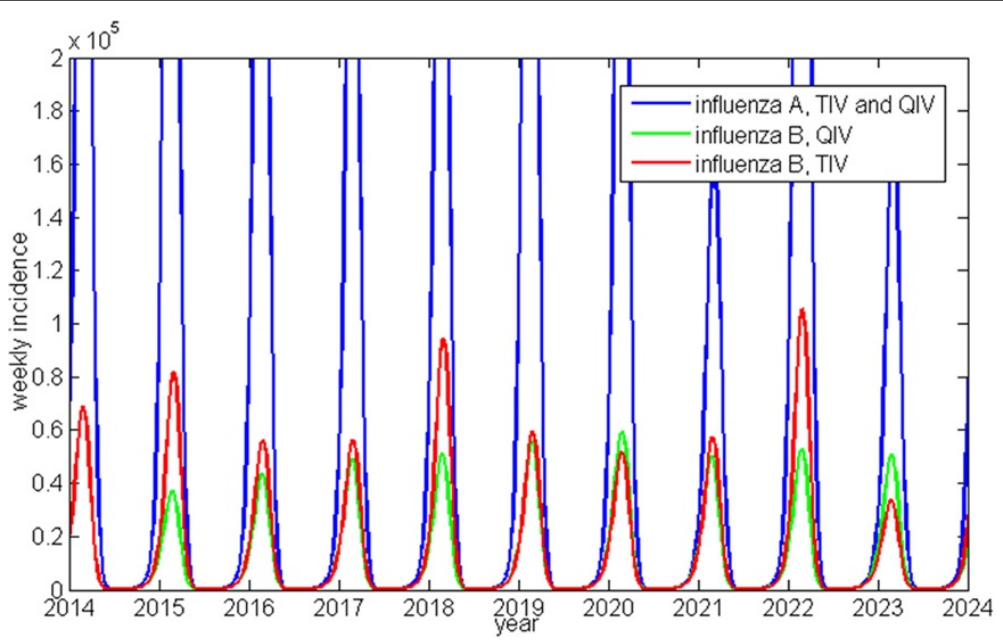

Fig. 1 Comparison of a typical TIV-QIV pair of model simulations (example of Canada). Note: The seventh and tenth influenza B peaks are actually slightly higher with QIV than with TIV. This is because the model is stochastic; TIV and QIV versions diverge so there is no real 1:1 correspondence between seasons after the first season in which QIV is introduced; Since TIV and QIV have identical efficacy against influenza A, the evolution of influenza A incidence with the TIV and QIV is identical between the pair of simulations 
A
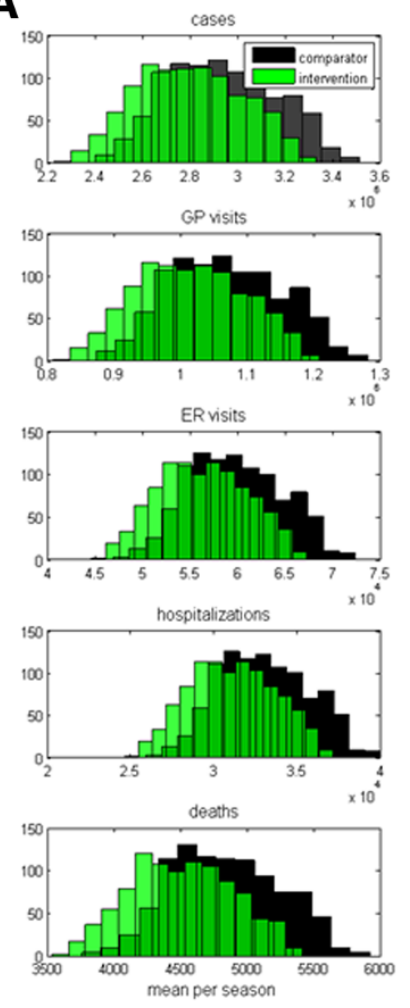

B
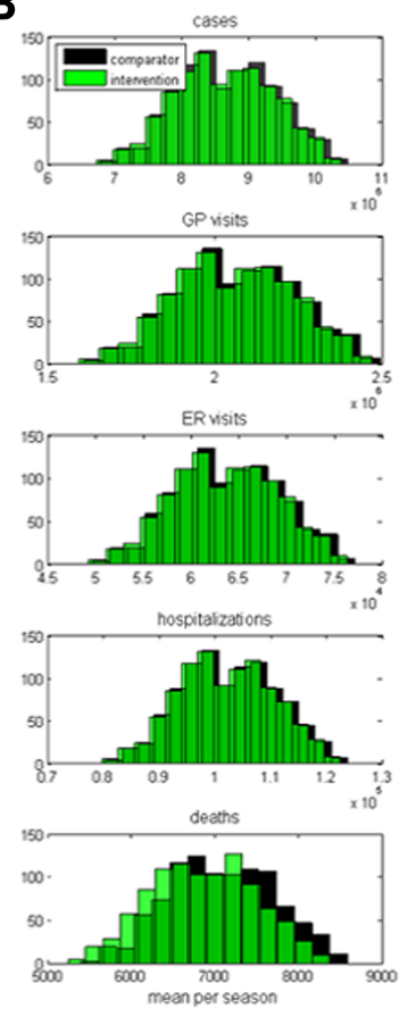
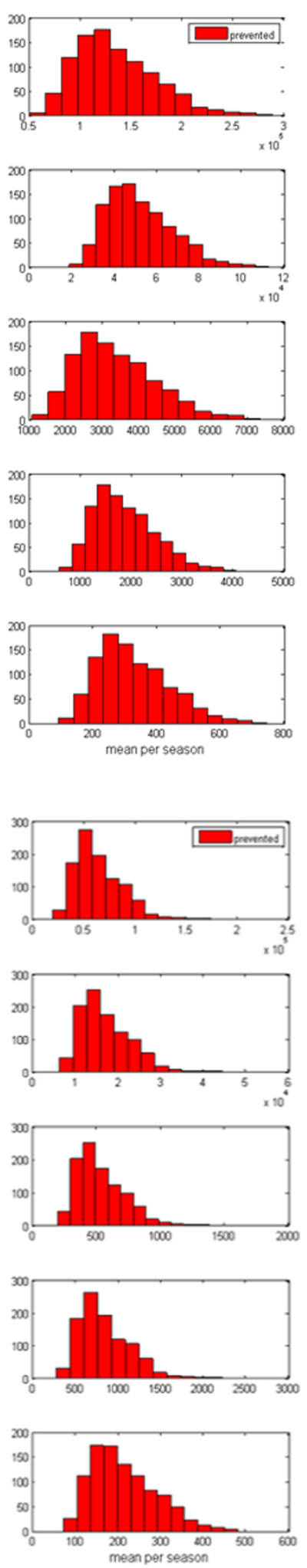

Fig. 2 Distribution of mean influenza-related outcomes per season (a) Canada (b) UK. Note: Outcomes are presented for TIV (comparator) and QIV (intervention) and mean outcomes prevented resulting from a switch from TIV to QIV (prevented) 
of death due to influenza rises sharply in individuals of ages above 65 years in both countries (Additional file 1, Table 1.1 and Table 1.2), thus the percentage of overall deaths prevented depends most strongly on the percentage of cases prevented in the elderly. Indeed, in both Canada and the UK scenarios, the percentage of cases prevented increases with age similar to vaccine uptake. This features more strongly in the UK where healthy individuals aged 18-64 years are not part of the public vaccination program.

A typical example of one pair of Canadian simulations, showing the impact of switching from TIV to QIV, is shown in Fig. 1. The distribution of model results for mean outcomes per season across all age groups with TIV, QIV and the difference between the two interventions per simulation pair, for Canada and the UK $(1,000$ model results each), are shown in Fig. 2.

\section{Cost-effectiveness analysis}

In Canada, discounting at $5 \%$, a switch from TIV to QIV would result in costs of $\$ 224, \$ 588, \$ 9,407, \$ 17,206$ and $\$ 10,1215$ per influenza case, GP visit, ER visit, hospitalization and death averted, respectively. Costs per QALY and life-year (LY) gained were estimated as $\$ 7,961$ (95 \% CI: \$3,080-\$16,320) and \$11,211 (95\% CI: $\$ 4,181-\$ 23,815$ ), respectively (Table 3 ).

For the UK (scenarios UK1/UK2), discounting at $3.5 \%$, a switch from TIV to QIV would result in costs of $£ 282 / £ 250, \quad £ 1,100 / £ 979, \quad £ 35,568 / £ 31,641, \quad £ 23,929 /$ $£ 21,207$, and $£ 117,428 / £ 110,496$ per influenza case, GP visit, ER visit, hospitalization and death averted, respectively. Discounted costs per QALY and LY gained were estimated at $£ 7,989$ (95 \% CI: 3,132-16,318)/£7,324 (95 \% CI: 2,937-14,044) and $£ 11,081$ (95 \% CI: 4,135-23,186)/£10,364 (95 \% CI: 4,029-20,588), respectively (Table 4).

The model results for the ICUR are presented as a cost-effectiveness acceptability curve, which shows, as a function of the adopted cost-effectiveness threshold, the probability that a switch from TIV to QIV would be cost-effective. For Canada, considering a threshold of $\$ 40,000-50,000$ per QALY gained [44], a switch from TIV to QIV is predicted to have a $100 \%$ probability of cost-effectiveness. For the UK, considering a threshold of $£ 20,000$ per QALY gained [57], the model predicts a probability of cost-effectiveness greater than $99 \%$ (Fig. 3).

\section{Deterministic sensitivity analyses}

Deterministic sensitivity analyses for both the countries indicate that among the model parameters varied, the modelled ICUR is most sensitive to the chosen range of QIV price per dose, outcome probabilities (varied simultaneously), to whether QALY loss due to non-fatal
Table 3 Modeled ICURs for Canada (discounted, $5 \%$ )

\begin{tabular}{ll}
\hline Category & Mean value $(95 \% \mathrm{Cl})$ \\
\hline Cost per case averted & $\$ 224(94 ; 419)$ \\
Cost per GP visit averted & $\$ 588(243 ; 1120)$ \\
Cost per ER visit averted & $\$ 9,407(3,678 ; 19,086)$ \\
Cost per hospitalization averted & $\$ 17,026(6,658 ; 34,546)$ \\
Cost per death averted & $\$ 101,215(37,429 ; 216,888)$ \\
Cost per QALY gained & $\$ 7,961(3,080 ; 16,320)$ \\
Cost per LY gained & $\$ 11,211(4,181 ; 23,815)$ \\
\hline
\end{tabular}

Note: GP, general practitioner; ER, emergency room; QALY, quality-adjusted life year; $L Y$, life year

influenza is taken into account, and to a hypothetical extreme case scenario in which breakthrough infections never result in a GP visit, ER visit, hospitalization or death, regardless of the type of vaccine used. For Canada, the ICUR varies from a minimum of $\$ 5,000$ per QALY (lower QIV price) to a maximum of $\$ 12,600$ per QALY (higher QIV price), while for the UK it varies from a minimum of $£ 4,000$ per QALY (high values for all outcomes rates) to a maximum of $£ 14,200$ per QALY (QALY loss due to non-fatal influenza neglected) (Fig. 4). Comparison to the accepted cost-effectiveness thresholds of $\$ 40,000-\$ 50,000$ per QALY gained and $£ 20,000$ per QALY gained in Canada and the UK respectively, suggests a high likelihood of cost-effectiveness in both countries, especially Canada, notwithstanding uncertainty in rates of outcomes and costs.

Table 4 Modeled ICURs for the UK (discounted, $3.5 \%$ )

\begin{tabular}{ll}
\hline Outcomes, $3.5 \%$ discount & Mean value $(95 \% \mathrm{Cl})$ \\
\hline UK1 & $£ 282(118 ; 538)$ \\
Cost per case averted & $£ 1,100(456 ; 2,113)$ \\
Cost per GP visit averted & $£ 35,568(14,729 ; 68,327)$ \\
Cost per ER visit averted & $£ 23,929(9,934 ; 45,494)$ \\
Cost per hospitalization averted & $£ 117,428(43,067 ; 246,342)$ \\
Cost per death averted & $£ 7,989(3,132 ; 16,318)$ \\
Cost per QALY gained & $£ 11,081(4,135 ; 23,186)$ \\
Cost per LY gained & \\
UK2 & $£ 250(105 ; 461)$ \\
Cost per case averted & $£ 979(404 ; 1,821)$ \\
Cost per GP visit averted & $£ 31,641(13,069 ; 58,892)$ \\
Cost per ER visit averted & $£ 21,207(8,909 ; 39,220)$ \\
Cost per hospitalization averted & $£ 110,496(42,719 ; 221,654)$ \\
Cost per death averted & $£ 7,324(2,937 ; 14,044)$ \\
Cost per QALY gained & $£ 10,364(4,029 ; 20,588)$ \\
Cost per LY gained &
\end{tabular}

Note: GP, general practitioner; ER, emergency room; QALY, quality-adjusted life year; LY, life year 

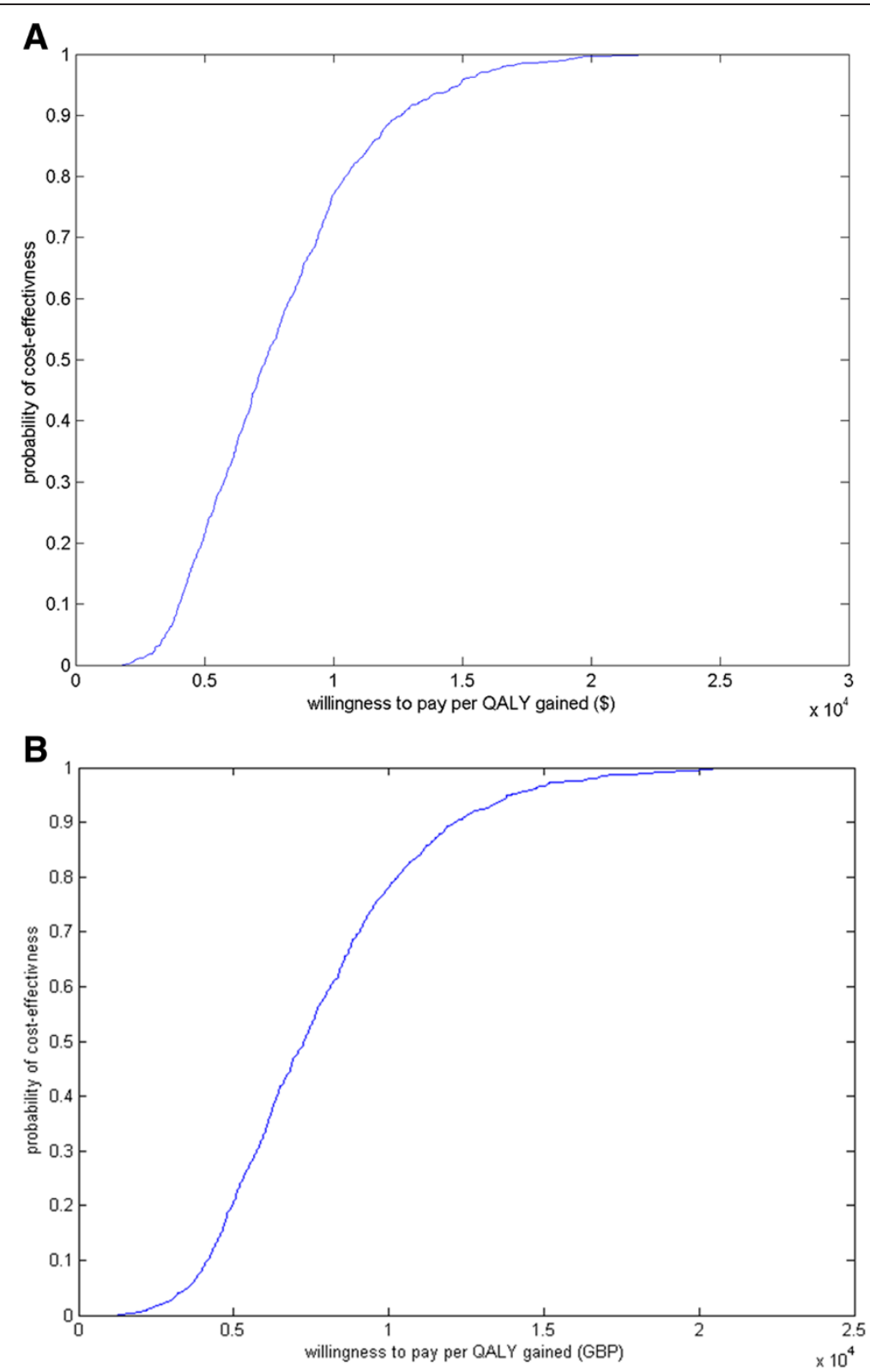

Fig. 3 Cost-effectiveness acceptability curves (a) Canada (b) UK. Note: QALY, quality-adjusted life-year; Cost-effectiveness threshold for Canada and UK are $\$ 40,000-50,000$ and $£ 20,000$ per QALY gained, respectively; ${ }^{\ddagger}$ Figure presented for the UK is based on Scenario UK1, UK2 is very similar (data not shown)

\section{Discussion}

This analysis aimed to assess the potential impact of switching from TIV to QIV over ten seasons in Canada and the UK. The dynamic transmission model in this analysis provides a detailed, age- and strain or lineagestratified representation of influenza dynamics, calibrated to surveillance data.

The model estimates that implementing a switch from TIV to QIV in both Canada and the UK would result in moderate reductions in the number of influenza cases and influenza-associated outcomes (GP and ER visits, hospitalizations and deaths). Though both countries have broadly similar demographic characteristics, we observed a significantly greater relative impact from the switch in Canada compared to the UK. The difference stems principally from vaccine uptake: While most Canadian provinces have universal influenza immunization programs (free vaccination for ages 6 months and above), the targeted vaccination program in the UK, although currently expanding to cover children, still leaves healthy adults aged 18-64 uncovered, resulting in significantly lower uptake in that age group as compared to Canada [33, 45]. 

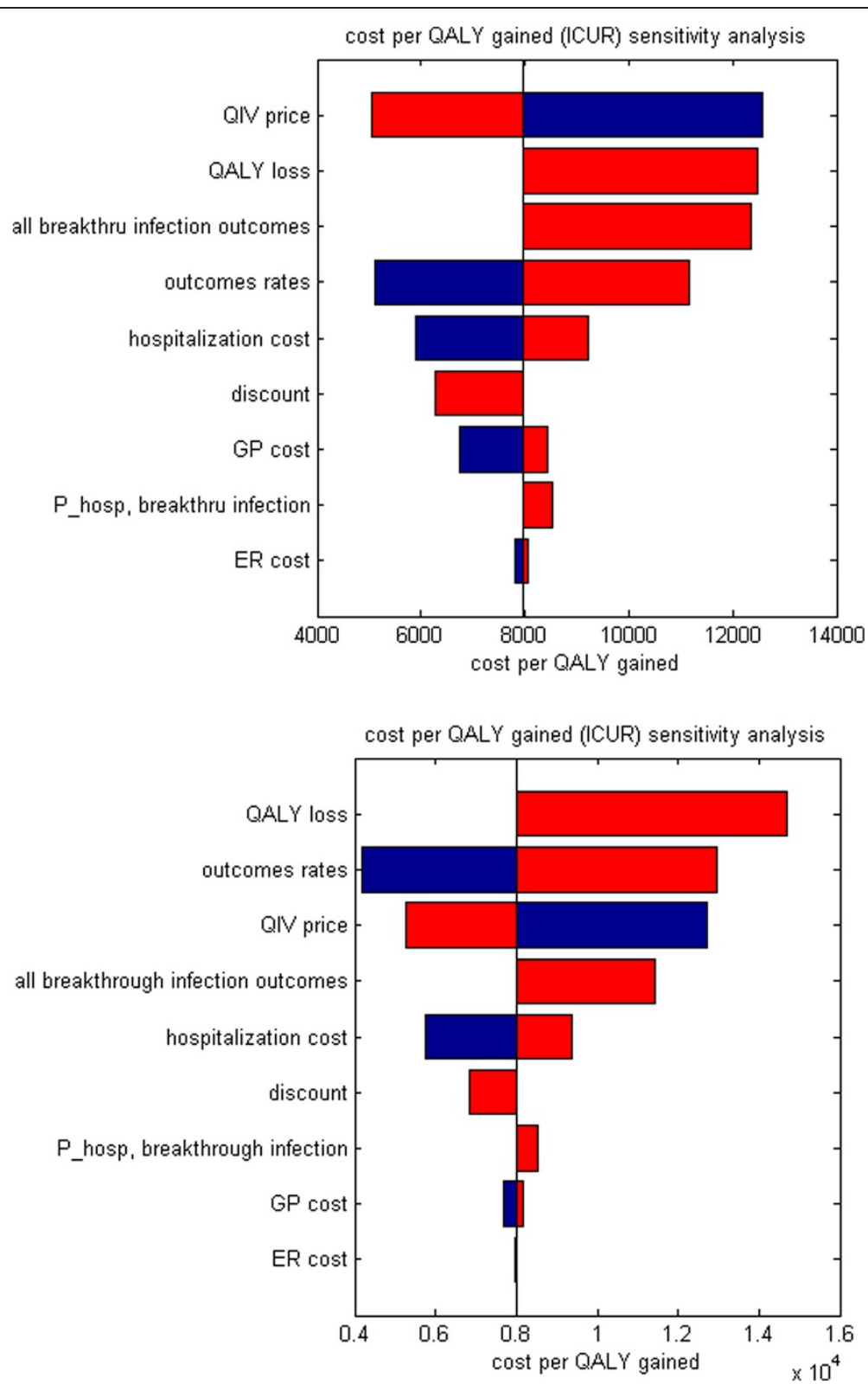

Fig. 4 Deterministic sensitivity analyses (a) Canada (b) UK $\mathbf{K}^{\ddagger}$. Note: The grey vertical line corresponds to all the uncertain parameters being at their respective base values. The red segments of the bars correspond to result values increasing the base case ICUR and the blue segments of the bars correspond to result values decreasing the base case ICUR; ${ }^{*}$ Figure presented for the UK is based on Scenario UK1, UK2 is very similar (data not shown); ER, emergency room; GP, general practitioner, QIV, quadrivalent influenza vaccine; QALY, quality-adjusted life-year

Despite a net increase in payer cost associated with the switch from TIV to QIV at the assumed QIV prices, the use of QIV is projected to be highly cost-effective in both countries, with an ICUR point estimate of $\$ 7,961$, $£ 7,989$ and $£ 7,324$ per QALY gained in Canada and in the two UK scenarios, respectively, far below each country's cost-effectiveness threshold. This conclusion is shown to be robust against uncertainty in the natural history parameters of influenza (as expressed in the cost-effectiveness acceptability curves, Fig. 4) in both settings, albeit more strongly so in Canada. Separate deterministic sensitivity analysis suggests it is also robust against uncertainty in costs and outcomes rates, again specifically in Canada.

A switch from TIV to QIV may carry additional benefits beyond those modeled here. Even though we have conservatively assumed that all vaccinated immunity only lasts one year on average, the slower antigenic drift of influenza B may result in some carry-over of influenza $B$ protection to subsequent seasons, thus further 
increasing the benefit of QIV relative to TIV [2]. Also, since vaccination with QIV is expected to result in fewer mismatched seasonal vaccine campaigns, its adoption may result in improved public perception of influenza vaccination, in turn translating into higher vaccine uptake [2].

Only a handful of studies have so far modeled the impact of switching from TIV to QIV on a country scale, in the US $[18,19]$ and the UK $[20,21,23]$. These studies have reported broadly similar findings to ours, of costeffectiveness across a broad range of QIV prices. However to our knowledge, there are thus far no published TIV-QIV studies utilizing a dynamic model, and none at all for Canada. Canadian studies of a different influenza vaccine intervention do exist as a basis for comparison, namely the transition in 2000 of the province of Ontario from targeted to universal immunization. A health economic study by Sander et al. [44], utilizing the results of an ecological study by Kwong et al. [58] (which served as a test case for the model we use here [26]), estimated the cost-effectiveness of Ontario's transition at $\$ 12,154$ per QALY gained. Our work here thus suggests that the cost-effectiveness of a TIV-QIV switch is comparable to that of a targeted-universal switch.

Our model was chosen for its ability to reproduce key elements of influenza dynamics: herd immunity, waning immunity, seasonality, age-specific contact patterns, and, critically for the problem studied here, the interplay of multiple influenza strains and influenza B lineages. However, like any model, it had limitations. Stratification of the population was only by age; healthy and at-risk parts of the population were not separately tracked. As described in [26], calibration of the natural history parameters of influenza was performed using the US as a setting, thus making the assumption that these differ negligibly between the US, Canada and the UK. It should be emphasized that the distribution of outcomes obtained through the calibration procedure (effectively a probabilistic sensitivity analysis on influenza natural history parameters) does not encapsulate uncertainty about the comparability of US to Canadian and UK influenza natural history. Due to the absence of Canadian data, US outcomes probabilities were used for Canada. Limitations in available LAIV influenza B match/mismatch efficacy data caused the LAIV and QLAIV modelled in the UK scenarios differ little in efficacy. Finally, we did not consider adverse events due to vaccination in this analysis. But given that both vaccines have similar safety profiles, this assumption is justified as it would not impact the model result.

\section{Conclusions}

Our findings predict that a switch from TIV to QIV is a highly cost-effective intervention to reduce the burden of influenza in both Canada and the UK, and thus suggest such a switch as a public health policy priority in both settings.

\section{Additional files}

Additional file 1: Model input data for Canada and UK.

(DOCX $53 \mathrm{~kb}$ )

Additional file 2: Age-specific mean health outcomes per season in Canada. (DOCX $51 \mathrm{~kb}$ )

Additional file 3: Age-specific mean health outcomes per season in the UK. (DOCX $65 \mathrm{~kb})$

\section{Abbreviations \\ $\mathrm{Cl}$ : confidence interval; $\mathrm{ClHI}$ : Canadian Institute for Health Information; ER: emergency room; GP: general practitioner; LAIV: live-attenuated influenza vaccine (trivalent); ICUR: incremental cost-utility ratio; LY: life-year; PSA: Probabilistic sensitivity analysis; QALY: quality-adjusted life-year; QIV: quadrivalent influenza vaccine; QLAIV: quadrivalent LAIV; TIV: trivalent influenza vaccine; UK: United Kingdom; US: United States of America; WHO: World Health Organization.}

\section{Competing interests}

ET, Al, and GM are employed by the GSK group of companies. Al and GM hold stock or stock options or restricted shares in the GSK group of companies. AC was employed by the GSK group of companies at the time of the study and is currently employed by Sanofi Pasteur. CTB received funding from the GSK group of companies to complete the work disclosed in this manuscript.

\section{Authors' contributions}

$\mathrm{Al}$ and $\mathrm{AC}$ contributed to the model development and calibration, the data acquisition and validation, the statistical analysis, and reviewed the study report. GM contributed to the development and selection of method and performed literature review. ET and CB performed literature review, reviewed the study report, contributed to the statistical analysis and to the model development and calibration. ET drafted parts of the manuscript. All authors had full access to the data, reviewed the manuscript, and gave final approval before submission.

\section{Authors' information}

ET is a member of the Waterloo Institute for Complexity and Innovation. His areas of research include biomathematics, economic and consumer behavior modeling, astrophysics and high-performance computing.

\section{Acknowledgements}

The authors would like to thank Vincent Laporte (Business \& Decision Life Sciences for GSK Vaccines) for editorial and publication coordination and Amrita Ostawal for medical writing (freelancer on behalf of GSK Vaccines). GlaxoSmithKline Biologicals SA was the funding source and was involved in all stages of the study conduct and analysis. GlaxoSmithKline Biologicals SA also funded all costs associated with the development and the publishing of the present manuscript.

\section{Author details}

${ }^{1}$ GSK, 7333 Mississauga Road, Mississauga, ON L5N 6L4, Canada. ${ }^{2}$ Department of Mathematics \& Statistics, University of Guelph, Guelph, Ontario, Canada. ${ }^{3}$ Department of Clinical Epidemiology and Biostatistics, McMaster University,

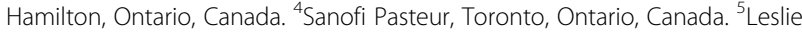
Dan Faculty of Pharmacy, University of Toronto, Toronto, Ontario, Canada. ${ }^{6}$ GSK Vaccines, Wavre, Belgium. ${ }^{7}$ Department of Applied Mathematics, University of Waterloo, Waterloo, Ontario, Canada.

Received: 15 April 2015 Accepted: 7 October 2015

Published online: 27 October 2015 


\section{References}

1. World Health Organization position paper on influenza vaccines. Wkly Epidemiol Record 2005; 33, 279-287.

2. Ambrose CS, Levin MJ. The rationale for quadrivalent influenza vaccines Hum Vaccin Immunother. 2012;8(1):81-8. doi:10.4161/hv.8.1.17623.

3. Daley AJ, Nallusamy R, Isaacs D. Comparison of influenza A and influenza B virus infection in hospitalized children. J Paediatr Child Health. 2000;36(4):332-5.

4. Hite LK, Glezen WP, Demmler GJ, Munoz FM. Medically attended pediatric influenza during the resurgence of the Victoria lineage of influenza B virus. Int J Infect Dis. 2007;11(1):40-7. doi:10.1016/j.ijid.2005.10.008.

5. Ng S, Cowling BJ, Fang VJ, Chan KH, Ip DKM, Cheng CKY, et al. Effects of oseltamivir treatment on duration of clinical illness and viral shedding and household transmission of influenza virus. Clin Infect Dis. 2010;50(5):707-14. doi:10.1086/650458.

6. Irving SA, Patel DC, Kieke BA, Donahue JG, Vandermause MF, Shay DK, et al. Comparison of clinical features and outcomes of medically attended influenza $A$ and influenza B in a defined population over four seasons: 2004-2005 through 2007-2008. Influenza Other Respir Viruses. 2012;6(1):3743. doi:10.1111/j.1750-2659.2011.00263.x

7. Prevention and control of seasonal influenza with vaccines. Recommendations of the Advisory Committee on Immunization PracticesUnited States, 2013-2014. MMWR Recomm Rep. 2013;62(Rr-07):1-43.

8. Tricco AC, Chit A, Soobiah C, Hallett D, Meier G, Chen MH, et al. Comparing influenza vaccine efficacy against mismatched and matched strains: a systematic review and meta-analysis. BMC Med. 2013;11:153. doi:10.1186/ 1741-7015-11-153

9. DiazGranados CA, Denis M, Plotkin S. Seasonal influenza vaccine efficacy and its determinants in children and non-elderly adults: a systematic review with meta-analyses of controlled trials. Vaccine. 2012;31(1):49-57. doi:10.1016/j.vaccine.2012.10.084.

10. Belshe RB. The need for quadrivalent vaccine against seasonal influenza. Vaccine. 2010;28 Suppl 4:D45-53. doi:10.1016/j.vaccine.2010.08.028.

11. Lo YC, Chuang JH, Kuo HW, Huang WT, Hsu YF, Liu MT, et al. Surveillance and vaccine effectiveness of an influenza epidemic predominated by vaccine-mismatched influenza B/Yamagata-lineage viruses in Taiwan, 201112 season. PLoS One. 2013;8(3), e58222. doi:10.1371/journal.pone.0058222.

12. Beran J, Peeters M, Dewe W, Raupachová J, Hobzová L, Devaster JM. Immunogenicity and safety of quadrivalent versus trivalent inactivated influenza vaccine: a randomized, controlled trial in adults. BMC Infect Dis. 2013;13:224. doi:10.1186/1471-2334-13-224.

13. Domachowske JB, Pankow-Culot H, Bautista M, Feng Y, Claeys C, Peeters M, et al. A randomized trial of candidate inactivated quadrivalent influenza vaccine versus trivalent influenza vaccines in children aged 3-17 years. J Infect Dis. 2013;207(12):1878-87. doi:10.1093/infdis/jit091.

14. Kieninger D, Sheldon E, Lin WY, Yu CJ, Bayas JM, Gabor JJ, et al. Immunogenicity, reactogenicity and safety of an inactivated quadrivalent influenza vaccine candidate versus inactivated trivalent influenza vaccine: a phase III, randomized trial in adults aged $>=18$ years. BMC Infect Dis. 2013;13(1):343. doi:10.1186/1471-2334-13-343.

15. Langley JM, Carmona Martinez A, Chatterjee A, Halperin SA, McNeil S, Keith S. Reisinger, Naresh Aggarwal et al. Immunogenicity and safety of an inactivated quadrivalent influenza vaccine candidate: a phase III randomized controlled trial in children. J Infect Dis. 2013;208(4):544-53. doi:10.1093/infdis/jit263.

16. Kim TH. Seasonal influenza and vaccine herd effect. Clin Exp Vaccine Res. 2014;3(2):128-32. doi:10.7774/cevr.2014.3.2.128.

17. Public Health Agency of Canada. Public Funding for Influenza Vaccination by Province/Territory (as of September, 2014). Available at: http:// www.phac-aspc.gc.ca/im/ptimprog-progimpt/fluvacc-eng.php. Accessed on: 1-Dec-2014.

18. Pebody RG, Green HK, Andrews N, Zhao H, Boddington N, Bawa Z, et al. Uptake and impact of a new live attenuated influenza vaccine programme in England: early results of a pilot in primary school-age children, 2013/14 influenza season. Euro Surveill. 2014;19(22):20823.

19. Public Health England. Flu Vaccination in Pilot Areas 2014/15. Stakeholder briefing, August 2014. Available at: https://www.gov.uk/government/ uploads/system/uploads/attachment_data/file/343878/ Stakeholder_briefing_14-15_F.pdf. Accessed on: 1-December-2014.

20. Lee BY, Bartsch SM, Willig AM. The economic value of a quadrivalent versus trivalent influenza vaccine. Vaccine. 2012;30(52):7443-6. doi:10.1016/ j.vaccine.2012.10.025
21. Reed C, Meltzer MI, Finelli L, Fiore A. Public health impact of including two lineages of influenza $B$ in a quadrivalent seasonal influenza vaccine. Vaccine. 2012;30(11):1993-8. doi:10.1016/j.vaccine.2011.12.098.

22. Van Bellinghen LA, Meier G, Van Vlaenderen I. The potential costeffectiveness of quadrivalent versus trivalent influenza vaccine in elderly people and clinical risk groups in the UK: a lifetime multi-cohort model. PLoS One. 2014;9(6), e98437. doi:10.1371/journal.pone.0098437.

23. Clements KM, Meier G, McGarry LJ, Pruttivarasin N, Misurski DA. Costeffectiveness analysis of universal influenza vaccination with quadrivalent inactivated vaccine in the United States. Hum Vaccin Immunother. 2014;10(5):1171-1180.

24. Tarride JE, Burke N, Von Keyserlingk C, O'Reilly D, Xie F, Goeree R. Costeffectiveness analysis of intranasal live attenuated vaccine (LAIV) versus injectable inactivated influenza vaccine (TIV) for Canadian children and adolescents. Clinicoecon Outcomes Res. 2012;4:287-98. doi:10.2147/ceor.s33444.

25. Postma MJ. Dynamic modeling for pandemic influenza. Expert Rev Vaccines. 2012;11(5):543-6. doi:10.1586/erv.12.30.

26. Thommes EW, Chit A, Meier GC, Bauch CT. Examining Ontario's universal influenza immunization program with a multi-strain dynamic model. Vaccine. 2014;32(39):5098-117. doi:10.1016/j.vaccine.2014.06.005.

27. Pitman RJ, White $L J$, Sculpher M. Estimating the clinical impact of introducing paediatric influenza vaccination in England and Wales. Vaccine. 2012;30(6):1208-24. doi:10.1016/j.vaccine.2011.11.106.

28. Vynnycky E, Pitman R, Siddiqui R, Gay N, Edmunds WJ. Estimating the impact of childhood influenza vaccination programmes in England and Wales. Vaccine. 2008;26(41):5321-30. doi:10.1016/j.vaccine.2008.06.101.

29. Marjoram P, Molitor J, Plagnol V, Tavaré S. Markov chain Monte Carlo without likelihoods. Proc Natl Acad Sci. 2003;100(26):15324-8. doi:10.1073/ pnas.0306899100.

30. Tully SP, Anonychuk AM, Sanchez DM, Galvani AP, Bauch CT. Time for change? An economic evaluation of integrated cervical screening and HPV immunization programs in Canada. Vaccine. 2012;30(2):425-35. doi:10.1016/ j.vaccine.2011.10.067

31. Van de Velde N, Brisson M, Boily MC. Modeling human papillomavirus vaccine effectiveness: quantifying the impact of parameter uncertainty. Am J Epidemiol. 2007;165(7):762-75. doi:10.1093/aje/kwk059.

32. Turner D, Wailoo A, Nicholson K, Cooper N, Sutton A, Abrams K. Systematic review and economic decision modelling for the prevention and treatment of influenza A and B. Health Technol Assess. 2003;7(35):iii-iv, xi-xiii, 1-170.

33. Government of Canada. Statistics Canada. Available at: http://www5. statcan.gc.ca/cansim/. Accessed on: 1-April-2014.

34. Office for National Statisitics. Available at: http://www.ons.gov.uk/ons/ index.html. Accessed on: 1-April-2014.

35. Zagheni E, Billari FC, Manfredi P, Melegaro A, Mossong J, Edmunds WJ. Using time-use data to parameterize models for the spread of close-contact infectious diseases. Am J Epidemiol. 2008;168(9):1082-90. doi:10.1093/aje/kwn220.

36. Mossong J, Hens N, Jit M, Beutels P, Auranen K, Mikolajczyk R, et al. Social contacts and mixing patterns relevant to the spread of infectious diseases. PLoS Med. 2008;5(3), e74. doi:10.1371/journal.pmed.0050074.

37. Molinari NA, Ortega-Sanchez IR, Messonnier ML, Thompson WW, Wortley

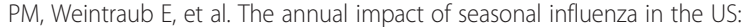
measuring disease burden and costs. Vaccine. 2007;25(27):5086-96. doi:10.1016/j.vaccine.2007.03.046.

38. Tappenden P, Jackson R, Cooper K, Rees A, Simpson E, Read R, et al. Amantadine, oseltamivir and zanamivir for the prophylaxis of influenza (including a review of existing guidance no. 67): a systematic review and economic evaluation. Health Technol Assess. 2009;13(11):iii. doi:10.3310/ hta13110. ix-xii, 1-246.

39. Meier CR, Napalkov PN, Wegmuller $Y$, Jefferson T, Jick H. Population-based study on incidence, risk factors, clinical complications and drug utilisation associated with influenza in the United Kingdom. Eur J Clin Microbiol Infect Dis. 2000;19(11):834-42.

40. Statistics Canada. Complete Life Table, Canada, 200-2002: females. Available at: http://www.statcan.gc.ca/pub/84-537-x/t/pdf/4198611-eng.pdf. Accessed on: 16-November-2014.

41. Statistics Canada. Complete Life Table, Canada, 200-2002: males. Available at: http://www.statcan.gc.ca/pub/84-537-x/t/pdf/4198612-eng.pdf. Accessed on: 16-November-2014.

42. Office for National Statistics, Interim life tables 2010-2012. Available at: http://www.ons.gov.uk/ons/publications/re-reference-tables.html? edition=tcm\%3A77-325699. Accessed on: 16-November-2014 
43. Mittmann N, Trakas K, Risebrough N, Liu BA. Utility scores for chronic conditions in a community-dwelling population. Pharmacoeconomics. 1999;15(4):369-76.

44. Sander B, Kwong JC, Bauch CT, McGeer A, Raboud JM, Krahn M. Economic appraisal of Ontario's Universal Influenza Immunization Program: a costutility analysis. PLoS Med. 2010;7(4), e1000256. doi:10.1371/ journal.pmed.1000256.

45. Moran K, Maaten S, Guttmann A, Northrup D, Kwong JC. Influenza vaccination rates in Ontario children: implications for universal childhood vaccination policy. Vaccine. 2009;27(17):2350-5. doi:10.1016/j.vaccine.2009.02.017.

46. Jefferson T, Rivetti A, Harnden A, et al. Vaccines for preventing influenza in healthy children. Cochrane Database Syst Rev. 2008;2, CD004879. doi:10.1002/14651858.CD004879.pub3.

47. Jefferson T, Di Pietrantonj C, Rivetti A, et al. Vaccines for preventing influenza in healthy adults. Cochrane Database Syst Rev. 2010;7, CD001269. doi:10.1002/14651858.CD001269.pub4.

48. Jefferson $T$, Di Pietrantonj $C$, Al-Ansary LA, et al. Vaccines for preventing influenza in the elderly. Cochrane Database Syst Rev. 2010(2):Cd004876. doi:10.1002/14651858.CD004876.pub3.

49. Jain VK, Rivera L, Zaman K, et al. Vaccine for prevention of mild and moderate-to-severe influenza in children. N Engl J Med. 2013;369(26):248191. doi:10.1056/NEJMoa1215817.

50. Jimenez-Jorge S, de Mateo S, Delgado-Sanz C, et al. Effectiveness of influenza vaccine against laboratory-confirmed influenza, in the late 20112012 season in Spain, among population targeted for vaccination. BMC Infect Dis. 2013;13:441. doi:10.1186/1471-2334-13-441.

51. Canadian Institute for Health Information. Patient Cost Estimator. Available at: http://www.cihi.ca/. Accessed on: 1-December-2014.

52. Skowronski DM, Woolcott JC, Tweed SA, et al. Potential cost-effectiveness of annual influenza immunization for infants and toddlers: experience from Canada. Vaccine. 2006;24(19):4222-32. doi:10.1016/j.vaccine.2005.12.036.

53. National Health Services. The Information Centre for Health and Social care. Prescription cost analysis, England 2011. Avaiable at: http:// www.hscic.gov.uk/catalogue/PUB05807/pres-cost-anal-eng-2011-rep.pdf. Accessed on: 1-April-2014.

54. British National Formulary. Available at: www.bnf.org. Accessed on: 1-April-2014.

55. Guidelines for the economic evaluation of health technologies: Canada [3rd Edition]. Ottawa: Canadian Agency for Drugs and Technologies in Health; 2006. Available at: http://www.cadth.ca/media/pdf/ 186_EconomicGuidelines_e.pdf. Accessed on: 8-April-2014.

56. National Institute for Health and Clinical Excellence. Discounting of health benefits in special circumstances. Available at: http://www.nice.org.uk/ article/pmg9. Accessed on: 8-April-2014.

57. Rawlins M, Barnett D, Stevens A. Pharmacoeconomics: NICE's approach to decision-making. Br J Clin Pharmacol. 2010;70(3):346-9. doi:10.1111/j.13652125.2009.03589.x.

58. Kwong JC, Stukel TA, Lim J, et al. The effect of universal influenza immunization on mortality and health care use. PLoS Med. 2008;5(10), e211. doi:10.1371/journal.pmed.0050211.

\section{Submit your next manuscript to BioMed Central and take full advantage of:}

- Convenient online submission

- Thorough peer review

- No space constraints or color figure charges

- Immediate publication on acceptance

- Inclusion in PubMed, CAS, Scopus and Google Scholar

- Research which is freely available for redistribution 